Meta

Journal des traducteurs

Translators' Journal

\title{
L'agestion ou la gestion en temps de crise
}

\section{René Deschamps}

Volume 29, numéro 4, décembre 1984

URI : https://id.erudit.org/iderudit/002547ar

DOI : https://doi.org/10.7202/002547ar

Aller au sommaire du numéro

Éditeur(s)

Les Presses de l'Université de Montréal

ISSN

0026-0452 (imprimé)

1492-1421 (numérique)

Découvrir la revue

Citer cet article

Deschamps, R. (1984). L'agestion ou la gestion en temps de crise. Meta, 29(4),

364-366. https://doi.org/10.7202/002547ar d'utilisation que vous pouvez consulter en ligne.

https://apropos.erudit.org/fr/usagers/politique-dutilisation/ 


\section{L'AGESTION \\ OU LA GESTION EN TEMPS DE CRISE}

RENÉ DESCHAMPS

Si cinq hommes peuvent creuser quatre tranchées en deux jours, combien en faudra-t-il pour en creuser 18 dans le même temps ?

Simple règle de trois au programme d'études du primaire et que les gestionnaires ont appris à utiliser tous les jours. Plus il y a de travail à faire, plus il faut de main-d'œuvre.

Pas malin, dira-t-on, c'est une opération purement mathématique. Mais les règles du jeu changent du tout au tout quand il s'agit de faire plus avec moins. La situation se complique, l'équation se trouve déséquilibrée. Courbes décroissantes, prévisions à la baisse, calculs «négatifs ", planification à rebours. C'est ce que j'appelle l'« agestion ».

La régression qu'on a subie ces dernières années a imposé au milieu des affaires une nouvelle approche dans la gestion des entreprises. Dans une société dite d'abondance, il était normal de recourir à des techniques administratives en fonction d'un accroissement constant de l'activité. Pendant des années, tout a monté. Il suffisait de prévoir les besoins en personnel et en matériel ainsi que les ressources financières, et tout tombait en place, comme par enchantement.

Mais il semble bien que l'époque des vaches grasses soit révolue et qu'il faille composer avec la récession qui a particulièrement frappé les services de traduction. Nonembauche et contraintes budgétaires sont maintenant monnaie courante.

Dans les lignes qui suivent, on verra l'évolution de la demande de traduction dans les grandes entreprises, à Montréal, ainsi que les bouleversements que la crise économique a provoqués dans la gestion des services.

\section{L'ÉVOLUTION DE LA DEMANDE}

La traduction dans les grandes entreprises de Montréal a connu une évolution marquée. Si pendant une quinzaine d'années on a assisté à une augmentation soutenue de la traduction de l'anglais au français, on constate maintenant non seulement une stabilisation de cette forme de traduction, mais une certaine diminution au profit de la traduction du français à l'anglais. Cette dernière, qui ne comptait que pour à peine $10 \%$ de tous les travaux il y a encore cinq ans, représente aujourd'hui $25 \%$ dans certains secteurs.

Malgré l'augmentation, dans des proportions gigantesques, de la documentation scientifique et technique, la traduction de ces publications ne s'est pas accrue au même rythme. Le phénomène tient en partie aux coûts élevés, et aussi au fait que l'entreprise, en général, réserve un meilleur accueil aux ouvrages étrangers venant des quatre coins du globe.

Changement aussi dans le genre de textes à traduire. Plusieurs entreprises, pour inciter leur personnel à rédiger davantage en français, se sont résolument mises à la tâche et ont à peu près éliminé complètement toute traduction de correspondance, d'avis 
de mutation et d'autres bulletins d'information à l'intention des employés. En revanche, la quantité des cours de formation semble avoir nettement monté.

Au total, il faut bien reconnaître que la demande de traduction, au lieu de fléchir comme on aurait pu le prévoir, s'est plutôt raffermie, au grand désespoir des services qui déjà ne peuvent suffire à la tâche.

\section{LA GESTION DU PERSONNEL}

Les techniques de gestion ont dû évoluer en fonction du ralentissement des affaires. De mathématicien qu'il pouvait être, le gestionnaire est devenu plus souple, plus attentif aux besoins du personnel. Presque plus humain. Il doit aller récupérer, tout au fond de lui, des aptitudes encore inexploitées (talents latents), des ressources qu'il soupçonnait à peine : imagination, esprit créateur, originalité. Le personnel est devenu, plus que jamais, la denrée rare par excellence. On veut retenir les bons éléments, de crainte qu'ils se mettent dans la tête de traverser la rue et d'offrir leurs services ailleurs.

On n'ose pas non plus faire maison nette et débarrasser le service du bois mort qui s'est accumulé avec le temps. On risquerait de se retrouver devant des postes vacants qu'on ne peut combler en raison de l'embargo rigoureux imposé sur l'embauche depuis quelques années déjà. Il est permis de se demander si certains chefs d'entreprise et directeurs de service ne se sont pas montrés indulgents ces dernières années envers le personnel marginal, préférant conserver chez eux des ressources médiocres plutôt que de risquer de tout perdre.

En temps de crise, les mots perdent leur sens. Le recrutement, par exemple, n'est plus ce qu'il était. Faire paraître des annonces dans les journaux, s'efforcer d'intéresser des employés qui travaillent ailleurs, interviewer des candidats, voilà des activités qu'on ne voit plus souvent, hélas!

Si la chaîne de production ne se renouvelle pas en partie au moins, si la machine ne se rajeunit pas, le vieillissement dégénère en sclérose. Il faut qu'il y ait périodiquement influx de sang nouveau.

Et les stagiaires d'été sont là pour nous le rappeler. Cette bouffée de jeunesse et d'air frais que certaines entreprises parmi les mieux nanties peuvent encore se permettre est un investissement des plus précieux.

Depuis quelque temps, malheureusement, les entreprises n'ont pas ouvert leurs portes aux étudiants comme elles le faisaient régulièrement. Le drame, c'est que les jeunes ne peuvent terminer leurs études, faute de moyens financiers suffisants et doivent s'engouffrer prématurément sur le marché du travail, pour bientôt venir grossir les rangs des prestataires d'assurance-chômage.

Les mutations, aussi, se font de plus en plus rares, et les promotions, pratiquement inexistantes.

\section{LE PLAN DE CARRIÈRE}

Le mot " carrière " n'a pas non plus le sens qu'on lui prête normalement. Les rares débouchés qui peuvent s'offrir sont des mutations dites "latérales", ne comportant ni promotion ni accroissement de privilèges. C'est le jeu de la chaise musicale. Pour le traducteur, les possibilités sont encore plus minces, car il a de la difficulté à se faire accepter au même titre que les autres cadres, sans doute en raison de l'allure d'artiste que certains se sont donnée. Ses chances d'avancement sont pratiquement nulles, à moins de pouvoir accéder à un poste de réviseur à l'intérieur même du service. En l'absence de vacance à ce niveau, il lui faut chercher ailleurs des tremplins. Et encore faut-il qu'il renonce à faire carrière dans la voie à laquelle il se destinait. S'il manifeste des qualités d'administrateur exceptionnelles, il pourra faire le saut dans un autre service et s'acclimater tant bien que mal à un régime de vie différent. Le professionnel devient alors gestionnaire. 
Tout comme le comptable et l'ingénieur. Et c'est bien ainsi. Il n'est pas dit que le traducteur y perdra au change. Au contraire.

\section{LA GESTION DE LA MACHINE}

Si on ne peut gonfler les effectifs, ni même combler les postes vacants, il reste heureusement une formule qui offre d'intéressantes perspectives : la machine.

L'expérience a démontré, dans les opérations de traduction d'un nombre de plus en plus grand d'entreprises, que les aides informatisées à la disposition des traducteurs et des réviseurs peuvent contribuer efficacement à augmenter la productivité. Le traitement de textes, notamment, qui se charge des tâches monotones et répétitives autrefois accomplies par les humains, a certes allégé le travail des traducteurs.

Parallèlement à ces aides, les "machines à traduire " ont envahi les milieux de la traduction. Après quelques essais plutôt infructueux, les gestionnaires ont dû les écarter presque définitivement. Indépendamment des bienfaits qu'ils peuvent rendre à l'entreprise, il reste que ces robots ne conviennent pas à la grande majorité des services de traduction, soit parce qu'ils sont offerts à des prix prohibitifs, soit parce qu'ils répondent mal ou pas du tout aux besoins actuels.

Les banques de terminologie, aussi, sont appelées à rendre d'immenses services parce qu'elles représentent la solution que recherchent les traducteurs depuis longtemps. Qu'elles appartiennent au secteur public ou privé, elles viennent combler une lacune que le milieu professionnel ne cesse de déplorer.

Si l'homme et la machine sont faits pour vivre en harmonie, espérons que l'agestion ne nous réservera pas, pour les années qui viennent, un bouleversement de l'ordre établi et qu'on ne retrouvera pas un jour à la tête des services de traduction, des machines disciplinées et efficaces, mais brutales et inhumaines. 